\title{
LA MEDIDA DE LA INCIDENCIA, PREVALENCIA Y PROBLEMAS CAUSADOS POR DROGAS ILEGALES
}

\section{Planteamiento del problema}

En los últimos veinte años, los problemas relacionados con el uso de drogas, y en todo caso la percepción social de los mismos, han creado una fuerte expectación que se ha traducido en una variada literatura con la pretensión de explicar las características, causas y consecuencias del fenómeno del uso de drogas.

La literatura surgida a partir de las drogas es de lo más variada y, en general, responde al modelo de transmisión de una moralidad que se supone debe impedir el «Consumo de Droga» entendido como una transgresión radical, una contaminación pecaminosa, que amenaza el orden e incluso la entropía del sistema. Es especialmente la literatura médico-biológica la que adopta este punto de vista $y$, obviando la posible objetividad del método científico, crea un tenebroso mundo de metáforas patológicas que llega, en ciertos ámbitos, a reemplazar otros infiernos. Una actitud similar aparece en otras disciplinas, aunque también se producen serios intentos de racionalización.

En las Ciencias Sociales, en cambio, han predominado modelos más «liberales» y permisivos, y el uso de drogas ha sido contemplado más bien como 
una conducta perseguida y reprimida, en cuyo contexto había que plantearse la defensa de la libertad y los derechos del individuo.

La Sociología ha sido la única oposición intelectual consistente frente a las valoraciones represivas y moralizantes de las supuestamente más neutrales "Ciencias de la Naturaleza», y durante varias décadas ha cumplido esta función con mayor o menor éxito.

Por su parte, la Antropología ha sido, y basta con mirar la obra de Gordon Wasson, el soporte teórico de las opciones «contraculturales», aunque sin involucrarse personalmente en tales movimientos. De todas formas, ambas ciencias sociales no son homogéneas y se han movido entre el «ensayismo liberal» y la investigación empírica, pareciendo esta última una aliada natural de las posiciones más represivas y moralizantes.

A partir de mediados de los años setenta, el modelo moralista comenzó a adquirir una clara hegemonía, situación de la que, por otra parte, siempre había gozado, y a imponer sus criterios a la hora de diseñar políticas «antidroga». Esta hegemonía la logró gracias al uso de los medios de comunicación, que le permitieron reforzar el modelo de la percepción social en sus aspectos más moralizantes y represivos, de tal manera que la propia sociedad, a causa de un obvio feed-back, ha producido las demandas de mayor control y represión. Sin embargo, al llegar a los años ochenta, este modelo conservador se ha visto claramente amenazado, mucho más de lo que lo fue por «la contracultura», y por un inesperado enemigo: la investigación sociológica empírica, que ha ido desarticulando cada uno de sus argumentos y ha comenzado a influir en grandes áreas de la opinión pública.

Esta modificación en la composición de fuerzas se evidencia por una serie de ejemplos claros, como son el cambio de actitud de los organismos internacionales, de la que fue pionera la Organización Mundial de la Salud, y en la que la planificación y normalización de la investigación sociológica ha pasado a ocupar el lugar estelar, o la de las Naciones Unidas, cuyo Boletín de Estupefacientes está empezando a prestar mucha atención a los trabajos sociológicos. Pero quizá el ejemplo más contundente lo constituyan los Estados Unidos, en donde se gestó el modelo moralista-represivo de Anslinger, y que en pleno auge del mismo, gracias a los varios millones de afiliados del Movimiento de Padres contra la Droga, pierde los papeles en uno de los más importantes documentos de una Administración norteamericana, la Estrategia Nacional contra las Drogas, 1985, diseñada por un Gobierno conservador, $y$ en el que por vez primera el modelo anslingeriano queda claramente fuera.

La razón de tal cambio aparece en el propio documento: la investigación sociológica empírica ha mostrado una realidad que con otras fórmulas más ético-filosóficas podía ser discutida, y sobre tal realidad se articulan las opciones y medidas. La Sociología ha pasado a ser, para los líderes del Movi- 
miento de Padres, de un enemigo al que combatir a un jefe al que es preciso manipular (Lindblad, 1983).

Al contrario, en Europa, con un menor desarrollo de la investigación empírica, aunque con una mayor fuerza de las corrientes liberales, los organismos paneuropeos se plantean seriamente una vuelta a las prácticas represivas y desde el Consejo de Europa llegan recomendaciones basadas en conceptos moralizadores.

La falta de datos empíricos supone que se crean modelos de la realidad, que se transmiten y utilizan con fines contrapuestos, dentro de una serie de fantasías, que ignoramos si servirán o no para cambiar el mundo, pero en ningún caso permiten contrarrestar las otras fantasías del modelo moralizante y represivo. Tenemos un ejemplo muy concreto de esta situación. En 1971, en el contexto de un estudio multinacional (Balter et al., 1974), las autoridades españolas comunican que en nuestro país han experimentado con ansiolíticos y sedantes un 10 por 100 de la población y que los usuarios son básicamente mujeres. Unos datos que llaman la atención a los autores del estudio, y no era para menos, porque unos años después las primeras encuestas muestran un nivel de experiencia del orden del 55 por 100 mínimo, y que la variable sexo era una función de la variable edad. ¿De dónde surgieron los datos españoles? De la pura especulación dentro de una lógica fantástica, que sirvió para dos argumentos contrapuestos: el de «el ama de casa drogadicta», de los posicionamientos liberales, y el de los jóvenes sólo drogas-drogas, de los posicionamientos conservadores. Una contraposición imaginaria que se limitaba al refuerzo de ambos discursos.

Evidentemente, los datos de base aportados por técnicas empíricas no lo resuelven todo, e implican una serie de problemas, tanto por las dificultades para obtener tales datos y su fiabilidad como por las limitaciones a su interpretación; pero tales problemas, a los que va precisamente dedicado este artículo, no significan que no se puede trabajar, sino que este trabajo tiene que ser cuidadoso y atento a las mediaciones metodológicas.

La investigación empírica sobre drogas se halla, además, en este momento, inmersa en una seria polémica en torno a su futuro, ya que, por un lado, aparecen diferentes metodologías con intención de mutua complementariedad $\mathrm{y}$, por otro, los sistemas de indicadores de prevalencia e incidencia, con una visión globalizadora y un coste que anula otras posibilidades.

En España, la investigación empírica tiene sólo un lustro de antigüedad, ya que no empezó hasta 1980 (Alonso Torrens, 1981), pero debido a la aceleración que se le ha imprimido al tema y a la gran cantidad de investigación desarrollada (Comas, 1985), en este momento se ha planteado ya el futuro y el dilema de crear o no un sistema de indicadores. Los sistemas de indicadores tuvieron un momento de esplendor en los EE. UU. en la década pasa$\mathrm{da}$, y se suponía que nos iban a proporcionar datos fiables y ajustados, aunque a un alto precio. Actualmente sólo está claro lo del coste. Pero existe una 
cierta polémica en torno a sus resultados que, en parte, se confunde con otros aspectos corporativos, ya que los indicadores suelen estar en manos de los sistemas sanitarios, en los que la presencia de científicos sociales es débil, mientras que otras metodologías pueden implicar más fácilmente a los científicos sociales.

Este es el planteamiento que pretende dilucidar este artículo, y para ello ha obviado parte del campo de investigación sobre drogas, en concreto la investigación sociológica sobre alcohol, tabaco y fármacos, ya que tales objetos exigen metodologías muy distintas (Seva, 1984; Generalitat de Catalunya, 1981) y los distintos niveles de investigación de cada droga exigen un desarrollo particular y complejo, por lo que nos vamos a centrar exclusivamente en las drogas ilegales, aunque se vayan a utilizar cuestiones relativas a alcohol, tabaco y fármacos en el propio desarrollo del discurso.

\section{Sistemas de notificaciones}

La puesta en marcha de un sistema de indicadores exige la existencia previa de un sistema de notificaciones eficaz, fiable y probado. Por sistema de notificación se entiende «un sistema de información basado en los datos suministrados a un organismo central de acuerdo con procedimientos de notificación sistemática» (Rootman, 1983). Los sistemas de notificación son, por tanto, equivalentes a los sistemas de estadística oficial, y en algunos casos, como los calificados de no-especializados, forman parte de los mismos. A través de un sistema de notificaciones podremos acceder a resultados paramétricos obtenidos de la información legal y formalmente prescrita e integrando todos los datos generados en la recogida de base.

Siguiendo a Rootman y Hughes, los objetivos de los sistemas de notificación son:

- Determinar la incidencia y la prevalencia del uso de drogas.

- Determinar las características de los usuarios conocidos.

- Medir permanentemente las tendencias y consecuencias del uso de drogas.

- Determinar cómo y en qué medida los organismos comunitarios son utilizados para combatir el abuso de drogas.

- Evaluar las acciones emprendidas para prevenir el uso de drogas.

En los últimos quince años, los sistemas de notificación desarrollados en diversos países han permitido obtener una cierta experiencia en cuanto a sus características metodológicas y a sus resultados. Rootman y Hughes los han clasificado según el tipo de notificación y según sean o no especializados. 
Los sistemas de notificaciones de episodios son aquellos que comunican al organismo central todos los episodios ocurridos en el centro comunicador, con independencia del número de individuos afectados o las veces que un mismo individuo resulta afectado.

El sistema de notificación de episodios especializados más conocido es el DAWN (Drug Abuse Warning Network), creado en 1972 por la Administración federal de los EE. UU. y basado en datos obtenidos en los servicios de urgencias hospitalarios y en el análisis de las muertes atribuidas al uso de drogas; los episodios que se recogen en los amplios formularios existentes son entonces las muertes y las incidencias que por uso de drogas se dan en los servicios de urgencias. Para rellenar el cuestionario existen en los servicios de urgencia dos «agentes cualificados» dedicados a esta misión.

Los formularios, una vez rellenos, se remiten al NIDA y éste obtiene así una estadística de episodios de urgencia y muerte relacionados con diversas variables como son las demográficas, tipo o tipos de droga, hábitos relacionados con el incidente, etc., hasta un total de 31. El sistema, de todas formas, es una muestra, ya que no cubre la totalidad del territorio de los EE. UU., sino sólo una serie de SMSA (Standard Metropolitan Statistical Areas), que empezaron siendo 39 y en los últimos años han llegado a 13.

Los objetivos del DAWN son muy ambiciosos y se resumen en:

- Identificar la amplitud del uso de drogas peligrosas para el individuo - la sociedad.

- Identificar las nuevas drogas o asociaciones de drogas que aparezcan en el mercado.

- Cuantificar la evolución de las tendencias del uso.

- Determinar las modalidades de uso.

- Evaluar los riesgos para la salud de tales usos.

- Recomendar la toma de medidas en función de la distribución social y espacial del uso de drogas.

Como ejemplo de un sistema no especializado de episodios podemos citar los sistemas policiales de información, que obtienen datos de detenciones de adictos o traficantes, operaciones emprendidas por la policía y cantidades decomisadas.

El segundo nivel es el de los sistemas de notificación de casos, que identifican a individuos concretos, aunque los afecten distintos episodios, siempre que ocurran dentro del mismo organismo notificador.

Como sistema de notificación de casos especializado hay que referirse al CODAP (Client Oriented Data Adquisition), creado al mismo tiempo que el DAWN para los centros de atención y tratamiento de drogodependientes y basado en cuatro formularios: 
1) Boletín de entrada.

2) Boletín de salida.

3) Boletín trimestral de actividades sobre cada paciente.

4) Resumen mensual del flujo de pacientes.

En cada centro existe un responsable designado por el NIDA y es obligatorio mantener unos ficheros permanentes con todos los datos contenidos en los formularios, parecidos a los del DAWN y en los que aparecen, además, datos relativos al tratamiento y sus resultados.

La identificación del paciente se consigue mediante el código asignado en el boletín de entrada, código que se mantendrá en los sucesivos boletines para el mismo paciente en el mismo centro por razones de confidencialidad. El NIDA posee, además, un sistema de control de la validez de los datos. En todo caso, no es un verdadero sistema de casos, ya que cada vez que un mismo paciente acuda a dos centros distintos se convierte en dos casos distintos.

Los ejemplos de sistemas no especializados de casos son muy abundantes, ya que así funcionan la mayoría de las estadísticas sanitarias, y en aquellas que aparecen desagregados los datos de uso de drogas conforman un sistema de notificación de casos.

Finalmente hay que considerar los sistemas de registro de casos, basados en la identidad del individuo, al que se sigue en su paso por los distintos organismos y episodios.

Los únicos sistemas de registro de casos especializados son los existentes en Inglaterra y en algunas de sus ex colonias. El Addicts Index (AI) inglés es un fichero de todos los drogodependientes identificados por las autoridades, sea en centros de tratamiento, hospitales o prisiones. Dicho fichero está en el Ministerio del Interior y, por supuesto, no es en absoluto confidencial, sino todo lo contrario: desde cualquier centro público se puede consultar al Ministerio en demanda de si una persona está fichada o no, incluso telefónicamente; en caso de que la consulta sea negativa, el personal médico o judicial que atiende al drogodependiente rellena el formulario y lo remite al organismo central. Dicho cuestionario se caracteriza por la cantidad de variables de identificación del sujeto; ejemplo: aparecen codificadas 39 características de su aspecto físico. El AI inglés, además, aparece desarrollado legalmente y obliga a su cumplimentación. 
Etapas de puesta en marcha de un sistema de notificaciones

1. PLANIFICACION INICIAL

- Determinar la necesidad del sistema.

- Precisar las finalidades.

- Definir los objetivos.

- Escoger el tipo de sistema.

- Determinar su viabilidad.

- Obtener la colaboración de las instituciones.

\section{CONCEPCION}

- Precisar los objetivos.

- Precisar los elementos de partida.

- Elegir los elementos de información.

- Establecer los formularios.

- Redactar las instrucciones.

- Elegir los organismos de notificación.

- Fijar los criterios de notificación.

- Definir los métodos de tratamiento de los datos.

- Definir los métodos de control de calidad.

- Definir los métodos de análisis.

III. PUESTA EN PRUEBA

- Ensayos prepilotos.

- Estudios pilotos.

IV. PUESTA EN MARCHA

- Asegurarse la participación continua de los organismos.

- Formar al personal.

- Mantener el sistema.

- Comunicar e interpretar los datos.

- Continuidad del sistema.

Fuente: Rootman, 1983.

Es también en el área de las ex colonias británicas donde aparecen sistemas no especializados de ficheros: existen registros de pacientes psiquiátricos obligatorios y centralizados en diversos países que, al desagregar los diagnósticos según la CIE, obtienen datos sobre drogodependientes y uso de drogas.

La puesta en marcha de un sistema especializado de notificaciones implica una serie de condiciones que se resumen en el cuadro adjunto, y que en ningún caso se han tenido en cuenta al construir los sistemas actualmente existentes en España, que surgieron exclusivamente a partir de un deseo de disponer de ciertos datos sin otra intención posterior.

Con independencia de los datos policiales, disponemos en España de un sistema de notificación de episodios no especializado, del cual sólo conocemos algunos resultados finales y sobre los que no parece posible actuar con el fin de extraer todas sus posibilidades, ya que el marco formal-burocrático no pa- 
rece pueda ser modificable. La Estadistica de la Fiscalía del Tribunal Supremo (Memoria anual del fiscal general del Estado) incluye el episodio de las intervenciones de los fiscales en caso de delitos contra la salud pública referidos al artículo 344 del Código Penal. Es un sistema muy poco útil en cuanto a los resultados, ya que sólo refleja el nivel judicial de la intervención (diligencias, sumarios y procedimientos) y la Audiencia implicada. No conocemos ni tan siquiera el número de individuos afectados, porque cada procedimiento puede agrupar a diversos individuos y los distintos procedimientos pueden afectar en varias ocasiones al mismo individuo o grupo de individuos. Menos útiles son aún el Movimiento Natural de la Población y la Encuesta de Morbilidad Hospitalaria, que, si bien utilizan la codificación de la CIE, no acceden adecuadamente ni a los fallecidos por uso de drogas ni a los hospitalizados por el mismo motivo.

\section{La construcción de indicadores y modelos matemáticos}

Una vez se dispone de un sistema de notificaciones se pueden construir indicadores y, a partir de los mismos, además, modelos matemáticos predictivos y explicativos. Vamos a exponer, como ejemplo, el indicador más desarrollado y evaluado, el HPI (Heroin Problem Index), que utiliza seis variables para medir la evolución del consumo de heroína en EE. UU. Dichas variables son (Person, 1977):

1) Admisiones de heroinómanos en programas de tratamiento.

2) Número de urgencias en hospitales relacionadas con heroína.

3) Muertes relacionadas con heroína.

4) Precio de la heroína en la calle.

5) Pureza de la heroína en la calle.

6) Cantidad de heroína decomisada.

La variable 1 se obtiene del CODAP; la 2 y la 3 , del DAWN; la 4,5 y 6 , de otras fuentes estadísticas de la DEA. Tales variables se refieren exclusivamente a una serie de SMSA elegidas en razón a la calidad de los datos aportados. Para cada una de ellas se obtiene un índice según la fórmula:

$$
\mathrm{HPI}=x 1 \cdot y 1+x 2 \cdot y 2+x 3 \cdot y 3+x 4 \cdot y 4+x 5 \cdot y 5+x 6 \cdot y 6
$$

para la que $x$ es el rango de cada SMSA para cada variable e $y$ el valor de cada una de las variables en cada SMSA. Mediante la introducción de supuestos de error se ordenan los SMSA, que se reordenarían en el caso de variar en los datos de las distintas variables, es decir, según la evolución de los resultados del DAWN y el CODAP, aunque no linealmente. Los cambios en 
el ordenamiento del HPI implicarían, así, modificaciones en la incidencia y prevalencia de la heroína.

¿Qué significa en realidad este indicador? Para Person y colaboradores, no existe ningún método para identificar o detectar las situaciones de dependencia, punto en el que hay una gran unanimidad, y para soslayar esta realidad acuden a una serie de supuestos (Person, 1976):

1) El uso de heroina es una conducta socialmente indeseable e ilegal que produce problemas al individuo, su familia y su comunidad.

2) La prevalencia del uso es directamente proporcional a la problemática.

3) Los sistemas de notificación recogen sistemáticamente las consecuencias de estos problemas.

Tales supuestos permiten renunciar al cálculo de los posibles dependientes y limitarse a plantear la existencia de un índice objetivo que se limita a cuantificar una evolución cuyos resultados determinan las necesidades de intervención. Aunque esto, por otra parte, no está demasiado claro en los textos, ya que las variables seleccionadas parecen haberlo sido más en función de la disponibilidad de los datos que de su significado. La progresiva reducción de SMSA apuntaría en el mismo sentido.

En el planteamiento original de 1976, además, Person y colaboradores habían introducido otras cuatro variables: dependientes detectados por encuestas, casos de hepatitis, arrestos por consumo de drogas y atracos a farmacias, que desaparecieron en la primera aplicación de 1977. Al año siguiente (Kozel, 1978), el HPI se limitó a una proyección de los resultados en cada una de las SMSA, sin cálculo del rango y la posición, que al ser comparadas con los datos de las encuestas sobre la población general y sobre la población escolar permitieron estimaciones del número de heroinómanos. Un dato que, en apariencia, la creación del HPJ desechaba.

Pero existen, además, otros modelos matemáticos multivariantes para medir la incidencia del uso de drogas ilegales como los desarrollados por Mulder, sea midiendo la probabilidad logística, sea midiendo la función probabilística directa y, en todo caso, a través de un juego matemático entre el riesgo, el suceso y la retirada obtenidos por las series temporales de las variables procedentes de los sistemas de notificación u otras series estadísticas (Mulder, 1986). En todo caso, ninguno de ellos se plantea ninguna duda en torno a los supuestos establecidos por Person, ni sobre la calidad de los datos de las variables utilizadas. 


\section{Los resultados de un esfuerzo}

Los sistemas de notificación permiten llegar a una serie de conclusiones muy fiables y contrastadas en relación a los objetos medidos, aunque quizá un tanto rodrejas en cuanto a su alcance. Recientemente ha aparecido una recopilación de los datos del DAWN y del CODAP, a la que se le han añadido otros datos procedentes de la Estadística Sanitaria del U. S. Department of Health, así como informes policiales (Kozel, 1985). Es relevante resaltar que éste es el esfuerzo de veinte años de mantener sin modificaciones costosos sistemas y que, a pesar de ello, los datos son incompletos, y los relativos a 1983 son ya sólo la extrapolación de ocho SMSA.

Veamos, en primer lugar, las siete conclusiones que figuran como tales y únicas en el informe, expuestas en forma resumida:

1. Entre $^{2} 1964$ y 1978, los EE. UU. sufrieron dos «Epidemias de He. roína», la primera se dio entre 1967-71 y la segunda entre 1974-76.

2." Hubo epidemias locales en 1979 (en Nueva York, con heroína procedente de Indochina) y en 1980-81 (en zona de Chicago, con heroína procedente de México).

3. ${ }^{a}$ En 1980, tres cuartas partes de los usuarios de heroína en tratamiento habían ya pasado por un tratamiento anterior, y la mitad de ellos llevaban más de ocho años de uso.

4. Los usuarios de heroína, incluidos los nuevos iniciados, eran en la década de los ochenta más viejos que los usuarios de las dos décadas anteriores.

5. Hasta 1972, el número de iniciados negros superaba al de blancos; a partir de este año la proporción cambió.

6." El abastecimiento de heroína depende de circuitos geográficos de difusión.

7. Las pautas de uso cambian para los nuevos iniciados en cada uno de los períodos epidémicos.

En segundo lugar transcribimos la Tabla de Resultados Globales de los distintos sistemas de notificación en las SMSA consideradas:

a Iniciados en el año en tratamiento.

$b$ Iniciados en el año.

c Casos de hepatitis B en hospitales.

d Pureza de la heroína en la calle.

$e$ Muertos por sobredosis de heroína.

$f$ Total usuarios de heroína (extrapolación nacional).

$g$ Número de tratados sobre un control en el CODAP de siete años. 


\begin{tabular}{|c|c|c|c|c|c|c|c|}
\hline Años & $a$ & $b$ & $c$ & $d(\%)$ & $e$ & $f$ & $g$ \\
\hline $1965 \ldots \ldots \ldots \ldots$ & 1.769 & 23.000 & & & & & \\
\hline $1966 \ldots \ldots \ldots \ldots$ & 2.081 & 43.000 & & & & & \\
\hline $1967 \ldots \ldots \ldots$ & 3.293 & 95.000 & & & & & \\
\hline $1968 \ldots \ldots \ldots \ldots$ & 4.890 & 158.000 & & & & & \\
\hline $1969 \ldots \ldots \ldots$ & 6.273 & 112.000 & & & & & \\
\hline $1970 \ldots \ldots \ldots \ldots$ & 5.596 & 284.000 & & & & & \\
\hline $1971 \ldots \ldots \ldots$ & 4.318 & 153.000 & & & & & \\
\hline $1972 \ldots \ldots \ldots$ & 4.186 & 205.000 & & & & & \\
\hline $1973 \ldots \ldots \ldots \ldots$ & 3.723 & 174.000 & & 5,1 & & 573.000 & \\
\hline $1974 \ldots \ldots \ldots$ & 3.776 & 178.000 & & 5,8 & 848 & 584.000 & \\
\hline $1975 \ldots \ldots \ldots \ldots$ & 4.023 & 179.000 & 13.121 & 6,1 & 1.234 & 540.000 & \\
\hline $1976 \ldots \ldots \ldots \ldots$ & 3.386 & 138.000 & 14.973 & 6,3 & 1.001 & 523.000 & \\
\hline $1977 \ldots \ldots \ldots$ & 2.854 & 109.000 & 16.831 & 5,3 & 417 & 495.000 & 14.746 \\
\hline $1978 \ldots \ldots \ldots \ldots$ & 3.240 & 55.000 & 15.016 & 4,4 & 366 & 471.000 & 11.770 \\
\hline $1979 \ldots \ldots \ldots$ & 3.316 & 80.000 & 15.452 & 3,6 & 337 & 420.000 & 17.174 \\
\hline $1980 \ldots \ldots \ldots \ldots$ & 3.171 & 63.000 & 19.015 & 3,8 & 440 & 492.000 & 22.438 \\
\hline $1981 \ldots \ldots \ldots$ & 3.150 & & 21.152 & 3,9 & 577 & & 20.659 \\
\hline $1982 \ldots \ldots \ldots \ldots$ & 3.330 & & 21.532 & 5,0 & 732 & & 18.862 \\
\hline $1983 \ldots \ldots \ldots \ldots$ & 3.230 & & 24.318 & 4,5 & 624 & & 16.767 \\
\hline $1984 \ldots \ldots \ldots \ldots$ & & & 16.440 & 4,7 & 844 & & 13.986 \\
\hline
\end{tabular}

\section{Todas las posibilidades como un proyecto de futuro}

La visión que acabamos de ofrecer podría llevarnos a concluir que la creación de los sistemas de notificación y la utilización de indicadores constituyen la metodología más adecuada para medir la incidencia, la prevalencia y los problemas causados por drogas ilegales, y que otras metodologías resultan inevitables y simples sustituciones por las dificultades de las diferentes Administraciones públicas nacionales para poner en marcha sistemas de notificación.

Pero esto no es así, ya que, por una parte, la utilización de indicadores produce una serie de dificultades y, por otra, el resto de metodologías socioepidemiológicas han tenido, en el campo específico de las drogodependencias, un notable desarrollo estos últimos años.

Incluso si tuviéramos que hacer caso a algunas recomendaciones de organismos internacionales nos encontraríamos con sorpresas, ya que la OMS acaba de recomendar en función del coste/eficacia (entendiendo por esta última la cantidad y fiabilidad de la información aportada) el siguiente orden de 
prioridades en la investigación sociológica sobre las drogodependencias (Rootman, 1985):

1) Cotejo de la información existente.

2) Estudios basados en informadores clave.

3) Estudios basados en observaciones.

4) Encuestas sobre la población general.

5) Encuestas específicas a grupos.

6) Sistemas de notificación.

El INSERM (Instituto Nacional de la Salud y la Investigación Médica de Francia) sostiene, muy razonablemente, que todo análisis empírico sobre el fenómeno del uso de drogas y las drogodependencias debe considerar dos facetas del mismo, la descriptiva y la analítica; la primera se refiere al conocimiento de los datos epidemiológicos y su desagregación en una red lo más fina posible de variables; la segunda, al análisis causal y la interpretación del fenómeno. Ninguna de ambas facetas puede llegar a resultados óptimos sin que exista un desarrollo paralelo de la otra (Davidson, 1982).

Un ejemplo claro de esta necesidad lo constituye el objetivo utópico de todos los investigadores en este campo: desvelar las condiciones de la «Ausencia de Riesgo» al tiempo que las características de los «Grupos de Alto Riesgo». Objetivo inalcanzable sin una dialéctica permanente entre la descripción cuantitativa y el análisis causal.

Por tal motivo hay que utilizar todas las metodologías posibles, ya que si bien unas nos aportarán cifras significativas, otras nos permitirán interpretaciones que facilitan la búsqueda de nuevas cifras, y viceversa, en el mismo sentido que se han desarrollado programas de investigación relativos a otras drogodependencias como son las Drogas Controladas tipo fármacos (Comité de Expertos, 1981), que resumimos en el cuadro siguiente de «Temas a Investigar»:

\section{1) Cálculo del suministro:}

- Producción (importación-exportación).

- Datos de ventas.

2) Evaluación y características de la distribución.

- Registros de prescripciones a población definidas.

- Registros de farmacias en ámbitos geográficos limitados.

- Archivos personales de médicos.

- Registros de instituciones que los administran directamente, como prisiones y hospitales. 


\section{3) Evaluación del consumo y sus características:}

- Encuestas generales a la población.

- Encuestas a grupos escogidos.

- Métodos cualitativos de campo.

- Historias de vida.

\section{4) Construcción de indicadores:}

- Especializados tipo DAWN y CODAP.

- No especializados a través de las estadísticas de mortalidad y morbilidad nacionales.

En resumen, alcanzar un conocimiento de la naturaleza y la amplitud del fenómeno, de su evolución y de los problemas asociados, de los factores de riesgo y de la evaluación de las intervenciones, a través de un sistema (similar a unas tablas input-output) en el que los distintos datos e interpretaciones actúen de mutuos elementos de control.

\section{Calcular los costes y evaluar las intervenciones}

Pero la investigación no puede ser contemplada sólo como una necesidad de conocimiento, sino que este conocimiento debe referirse siempre a unos costes y a las ventajas que se puedan derivar de la aplicación de los mismos. Un objetivo quizá utópico, que no debe nunca coartar la libertad personal ni limitar la posible prospección, pero que cada vez es más necesario, y no precisamente de cara a los «pequeños investigadores», sino en las grandes organizaciones de investigación.

En el campo de las drogodependencias, el coste de los sistemas de notificación y de los indicadores no parece justificar los resultados obtenidos por los mismos; en cambio, otro tipo de trabajos más simples nos aportan mayor información.

Tomemos el caso, casi paradójico, de sistemas de evaluación del coste y resultados terapéuticos de las distintas intervenciones. El NIDA ha puesto en marcha tres sistemas distintos para cubrir este objetivo.

Tenemos, en primer lugar, el DARP (Drug Abuse Reporting Programme), que sobre una muestra de centros y sus resultados, sobre el que aplica luego un indicador, llega a determinar si las comunidades terapéuticas (CT), los mantenimientos con metadona (PMM) o las terapias ambulatorias (TA) obtienen mejores resultados y a qué precio (Sells, 1979). Las carencias metodológicas del DARP aconsejaron la creación del TOPS (Treatment Outcome Prospective Study), que es una muestra en panel de individuos pasados con éxito por un tratamiento, en los mismos tres niveles que el DARP y con los 
mismos objetivos (Tims, 1984). Tanto el DARP como el TOPS mantienen una costosa infraestructura en una serie de centros de atención y una red permanente de controladores de los individuos del panel.

Un tercer sistema es el NYPS (National Youtb Polydrug Study), una encuesta sobre una muestra de población atendida en los distintos centros que establece las características de los atendidos y la influencia de éstas en el resultado del tratamiento por tipo de centro (Santo, 1980). El coste es el de una encuesta.

¿Hasta qué punto los sistemas DARP y TOPS justifican su coste en relación al NYPS? Creemos que de ninguna manera, ya que hasta ahora sólo han podido demostrar que los programas de mantenimiento con metadona proporcionan resultados de estabilidad personal similares a los que la abstemia e integración logrados en las comunidades terapéuticas o los tratamientos ambulatorios. Lo cual, por otra parte, tampoco ha significado la reducción del volumen de los PMM en EE. UU. El NYPS, además, ha proporcionado una serie de correlaciones entre éxito del tratamiento y características del tratado, aunque parece que esto es también posible utilizando los formularios obtenidos por el DARP y trabajando sobre ellos como si se tratara de una muestra para establecer correlaciones entre las variables sociodemográficas y los resultados de un tratamiento, con la importante ventaja de que la muestra puede ser muy manipulable, y hasta aislar cohortes concretas (Joe, 1978).

Pero, por otra parte, ¿en qué se diferencian los resultados de estos grandes programas de evaluación con los programas locales? Recientemente, dos autores españoles nos han ofrecido un panorama de los trabajos de seguimiento realizados por los propios centros o por las autoridades locales de las que dependen los individuos tratados (Guardia y González, 1983). Pues bien, estos autores confirman que, en conjunto, estos microestudios, realizados con medios muy precarios, no sólo of recen resultados al mismo nivel que el DARP, TOPS y NYPS, sino que, además, manejan un volumen de variables mucho más amplias que éstos. Aunque en todos los casos, y de ello nos ocuparemos en el próximo apartado, estas variables posean un significado limitado.

Pero la cuestión de los costes no acaba aquí, porque es preciso introducir otra cuestión como es la razón para introducir la intervención, incluida su evaluación. Existe una fuerte tradición de estudios sobre los costes económicos del uso de drogas (Rufener, 1976), pero éstos son sólo los medibles en términos monetarios, es decir, a aquellos que se les puede asignar una traducción, normalmente de la carencia de actividad o del coste de la intervención, medible en moneda corriente (Simon, 1982).

El tema es aquí la selección de las traducciones. La heroína siempre resulta seleccionada, y los factores asociados al coste de la heroína significan una larga relación; sin embargo, los costes de otras drogas o no son nunca seleccionados o lo son con una relación de factores más reducida.

En España se ha evaluado en diversas ocasiones el coste económico de la 
heroína con resultados asaz espectaculares, ya que se ha llegado a incluir la pérdida del coste público de los estudios realizados por el drogodependiente no insertado; en cambio, los costes económicos del tabaco, recientemente calculados por vez primera, son inferiores, ya que sólo se han contemplado los costes para la Seguridad Social de una parte de las enfermedades derivadas del hábito de fumar. Incluso algo tan obvio como las Incapacidades Laborales Transitorias, la Larga Enfermedad o la Invalidez Permanente a causa de enfermedades producidas por el tabaco no han sido incluidas.

Viene esto a raíz de que los sistemas de notificación, los indicadores y otros sistemas de evaluación muy costosos quizá no se aplican a medir cuestiones cuyo coste real justificaría los desembolsos, sino a problemas de orden inferior pero sobre los que existe una determinada presión social, que explicaría, además, por qué gastar tanto dinero en este tipo de investigación.

\section{La supervivencia de las encuestas}

En los apartados anteriores reaparecen las encuestas, cuando se suponía que los sistemas de notificación, al ser exhaustivos, evitaban la necesidad de obtener resultados procedentes de muestras representativas. Pero esto no ha sido así por dos razones fundamentales: la primera, de la que nos ocuparemos en el próximo apartado, porque los sistemas de notificaciones sólo incluyen cierto tipo de datos $y$ carecen de representatividad a pesar de su exhaustividad, y la segunda, porque los datos obtenidos por los sistemas de notificación y los indicadores construidos a partir de los mismos son mucho menores que los obtenidos a partir de encuestas.

Podemos comparar el DAWN y el HPI con los resultados del panel que mediante encuestas a la población escolar realiza anualmente, desde 1975 , el Institute for Social Research, de la Universidad de Michigan, del que recientemente se han publicado dos recapitulaciones (Johnston, 1984 y 1985).

La tasa de resultados que arroja dicho panel no nos permite hacer un resumen del mismo, pero entre el agobio de conclusiones se pueden extraer algunas que adquieren mayor relevancia desde el momento en que no fueron detectadas a través del sistema de notificaciones e indicadores: la caída espectacular de las tasas de incidencia de la heroína entre los adolescentes, el incremento de la incidencia y prevalencia de la cocaína, el ligero descenso de la prevalencia de la cannabis, la existencia de ciclos cerrados en el caso del PCP o los inhalantes, los cambios de actitudes, pautas y opiniones ligadas a estos nuevos fenómenos, etc.

Sin duda, el panel de Michigan reúne unas características (una muestra de 12.000 a 18.000 escolares, con seguimiento independiente en cada cohorte) que explican la espectacularidad de los resultados. Pero el mismo director del proyecto publica en otro texto (Johnston, 1980) un resumen de la metodología 
y resultados de 38 encuestas sobre la población escolar realizadas en países avanzados y en otros del Tercer Mundo como India, Perú, Indonesia o Irán, con una conclusión interesante: los resultados obtenidos por un método como el de encuesta dependen básicamente de la tradición y la experiencia del país en encuestas sobre drogas y del número y profesionalidad de redes de campo dedicadas al muestreo.

Quizá uno de los problemas de las encuestas era la falta de homogeneidad en los resultados y una cierta espontaneidad en los planteamientos, hasta el punto que las quejas sobre esta cuestión son permanentes: «los datos obtenidos se refieren a fenómenos distintos» (Johnston, 1980); «las encuestas no describen los mismos fenómenos» (Hughes, 1983). Pero teniendo en cuenta la experiencia de Michigan, la Organización Mundial de la Salud ha propuesto una serie de cuestionarios que han concluido en dos modelos (con posibilidad de seleccionar grupos de items), el uno para jóvenes estudiantes (Smart, 1980) y el otro para jóvenes en general (Smart, 1981).

Ambos cuestionarios están siendo utilizados masivamente, sin que España constituya una excepción, y en este momento el número de aplicación en nuestro país puede rebasar el centenar, de ellas una treintena ya publicadas. Esta homologación permite comparaciones nacionales e internacionales, así como el mantenimiento de una especie de «panel no programado» de datos.

Pero, además, la propia OMS ha puesto en marcha un programa de proyectos-piloto con el único fin de perfeccionar los modelos propuestos (Hughes, 1983). En tales proyectos aparece una distribución de rúbricas de base, preguntas e items ya codificados que nos van a permitir una selección sobre todas las posibilidades imaginables, a partir de la que podremos construir cualquier cuestionario sin perder las ventajas de la comparación y el panel.

Por otra parte, el inconveniente de la falta de continuidad de las encuestas, expuesto por los partidarios de la utilización de indicadores (Rootman, 1983), no es una consecuencia del método utilizado, sino que depende de decisiones administrativas. La misma decisión que puede optar por cerrar o mantener un sistema de notificación vale para decidir si se hace o no una encuesta. Sin duda, toda decisión administrativa viene muy condicionada por los costes de la investigación, hasta el punto que parece preferible proponer paneles de encuesta en vez de indicadores para obtener "datos más estables» (Smart, 1982).

Las ventajas de las encuestas son innegables, y más si se tiene en cuenta sus costes. Un resumen de ventajas y desventajas aparece en algunas publicaciones (Smart, 1980; Johnston, 1984), pero una de las desventajas es significativa: las encuestas sobre drogas no son verdaderamente significativas nunca. Existe, como en el caso de los sistemas de notificación, una selección previa de audiencias que inhibe la propia voluntad de una representación general. Aparentemente, la segregación de audiencias actúa muy fuertemente 
en el caso de las drogas (Comas, 1981), y la selección previa aumenta la fiabilidad. De ahí la insistencia en hacer encuestas a estudiantes.

Pero, además, está la cuestión del secreto ligado a conductas expresivas, sobre el que insistiremos más adelante, y que produce una serie de dificultades en el manejo de los datos procedentes de encuestas.

Estos problemas han intentado ser solventados sin mayores modificaciones metodológicas con una serie de originales trucos, como el realizar paneles con entrevistados escolarizados cuando dejan de estarlo, con resultados muy interesantes en la correlación de las variables residenciales, laborables y familiares (Bachman, 1984), o como el Proyecto Multiciudades del Consejo de Europa, que pretende construir una especie de indicador partiendo de sólo los datos de «haber experimentado» obtenidos mediante encuesta a estudiantes en varias capitales europeas (Driessen, 1985).

Los métodos para controlar el secreto e identificar los dependientes, cosa que no parece cuantitativamente posible (Comas, 1985), son más sofisticados y van desde la introducción de test de ansiedad en paralelo (Mohan, 1980) a la doble consulta con preguntas-filtro cruzadas (Medina-Mora, 1981).

\section{Los restos del naufragio en la isla de Robinson}

Tras el conjunto de investigaciones empíricas que venimos describiendo existen una serie de supuestos, tan rígidos como los de Person o tan limitativos de la población considerada como universo como los de Johnston, y que, en general, atribuyen a la percepción social la categoría ontológica de determinante de las posibles cuestiones a plantear. Esto produce muchas dificultades a la hora de valorar los problemas relacionados con el uso de drogas, y a ello le vamos a dedicar el próximo epígrafe; pero, como hemos indicado en el anterior, existen, además, una serie de problemas de representatividad que nos toca dilucidar ahora.

Los sistemas de notificaciones y los indicadores se limitan a identificar al individuo concreto: el «heroinómano» sometido a procesos de intervención. $\mathrm{Y}$ este «heroinómano» es, además, un individuo indefinido como usuario dependiente de drogas mientras haya experimentado en diversas ocasiones con heroína. Está en situación mórbida en sentido estricto: «desviación subjetiva u objetiva de un estado de bienestar fisiológico» (Comité de Expertos en Estadística de la OMS), pero no tiene que ser necesariamente un dependiente farmacológicamente determinado.

Pero este «heroinómano» establece una pauta, un límite a la indagación, un espacio real pero con unos límites fantásticos, que hacen de los datos otra pura fantasía. Se supone que las notificaciones recogen al menos la incidencia del uso de heróna, pero los estudios demuestran que la media del primer 
contacto con instituciones de intervención es de treinta y seis meses después de la primera experiencia (Ingold, 1982). ¿Cómo medir así la incidencia?

En un texto anterior (Comas, 1981) establecía que la investigación psicosocial sobre «intervenidos» presentaba un doble sesgo: por un lado, el motivo de la intervención $y$, por el otro, el tipo de centro, y llamaba la atención sobre la peligrosa tendencia, que luego se ha generalizado, a utilizar los datos de tales investigaciones como si se tratara de la realidad sociológica sobre las drogas. La propuesta, en aquella ocasión, se refería a la realización de trabajos puntuales sobre grupos definidos de «intervenidos» sobre los que fuera posible establecer comparaciones en base a un protocolo común que permitiera: a) una clara identificación sociodemográfica y socioeconómica; b) una integración de los resultados del comportamiento de las variables sólo en función de tal identidad.

Aparentemente, éste es un hecho que se acepta sin discusión, pero si observamos algunos de los estudios empíricos españoles, los más consistentes (como los de Obiols, 1980; Monegal, 1980; Ramón, 1980; Villa-Abadal, 1983, y Rodríguez-Martos, 1980), veremos que todos hablan de muestra y llegan a conclusiones sobre los usuarios de drogas. Esto es aún más evidente en el -excelente trabajo, por otra parte- texto de Díaz (1985), que resume toda la cuestión, y en el que aparece como motivo de consulta la heroína en un 80,4 por 100 y la cocaína en un 0,5 por 100 ; lo que no deja de ser cierto, pero eso no significa que éste es el nivel real ni tan siquiera de la demanda o de los problemas causados por cada una de las respectivas drogas.

Los datos procedentes de un grupo de atendidos sólo reflejan las características del grupo y no poseen significado sobre el conjunto de la población de usuarios. Las opiniones de los profesionales de la intervención reflejan sólo tales opiniones y no las características objetivas de la población atendida. Tales obviedades no son en la práctica asumidas metodológicamente por el planteamiento de los sistemas de notificación, que producen unos resultados limitados con pretensión de universalidad.

Así, un sistema de notificación no ofrece datos ni de incidencia ni de prevalencia, punto que comparten los profesionales que lo establecen; pero los indicadores que pretenden medir la evolución de unos parámetros abstractos, pero significativos, tampoco valen porque se hallan sometidos a la influencia de una serie de factores que modifican los resultados del índice con independencia de la evolución real de la prevalencia.

Aparte hay que mencionar que el sentido abstracto del índice no es transmitido así a la opinión pública; es que incluso los profesionales no socioepidemiológicos lo utilizan como valor absoluto con significado real. Pero ¿cuáles son estos factores que influyen en los resultados de los indicadores sin modificar la realidad? Tomemos unos ejemplos, empezando por el caso de PCP.

EI DAWN empieza a detectar «la incidencia» de dicha droga y se ponen en marcha todos los mecanismos, tanto de control policial como de alarma 
social, así como de prevención y atención prioritaria, y a partir de ahí la droga desaparece. Resulta que el PCP ha sido utilizado y distribuido a un sector muy delimitado de adolescentes con poca experiencia, a través de una cierta promoción en una prensa «musical» muy especial. Es, además, una droga cuyas consecuencias son muy complejas y difícilmente controlables. Resultado: los servicios de urgencia empiezan a encontrarse con «intoxicaciones agudas» por PCP. Conclusión del DAWN: se trata de una nueva epidemia, una droga con un nivel de uso similar a la cannabis o la heroína, cuando en realidad apenas es usada por una exigua minoría y en unos cortos y cíclicos períodos de tiempo (Feldman, 1980).

Otro caso podemos observarlo cuando las muertes asociadas con los preparados de heroína inyectados en la calle se incrementan sustancialmente en el distrito de Columbia, entre abril de 1979 y diciembre de 1982. La tasa de mortalidad en 1981, 17,4 por 100.000, es posiblemente la más alta nunca registrada. Un estudio de caso-control basado en los análisis posmorlen de muestras de sangre indica que la concentración de heroína y etanol son factores de riesgo en las muertes relacionadas con la heroína. El análisis de la composición de calle-nivel de preparación de heroína y la mortalidad trimestral indica que la cantidad de heroína en paquetes vendidos en la calle, el precio de la heroína en estos paquetes y el peso de la quinina por cada paquete predice, asimismo, las muertes. Un incremento en el uso casual de la heroína en combinación con etanol y quinina es la causa probable de este incremento en el número de muertes, pero no un cambio en las cifras de prevalencia de heroína (Ruttenber, 1984).

Podemos ver un caso más cercano en el hecho de que los sociólogos se han limitado a analizar la evolución de los datos de «causa de muerte» en la CIE sin tener en cuenta que dicha evolución, de hecho, tenía mucho más que ver con la conceptualización de las causas que se hacían en el colectivo médico en relación a los cambios ideológicos y teóricos que con la realidad objetiva de evolución de las causas de muerte.

Tenemos el ejemplo de que las muertes atribuidas al alcohol se vienen incrementando desde 1950 , en un porcentaje medio del 7 por 100 sobre el año anterior. Al finalizar la década, proyectando la tendencia, el porcentaje sobre el total de fallecimientos puede superar un 7 por 100 , habiendo partido de menos de un 1 por 100. Sin duda, entre 1950 y 1986 el consumo de alcohol se ha incrementado considerablemente y puede haberse duplicado. Pero en el mismo período las muertes atribuidas al alcohol se habrán sextuplicado. La causa hay que buscarla en un incremento de la conciencia del colectivo médico hacia la «causalidad alcohólica», y a su reflejo en la «causa de falleci. miento» del certificado de defunción. 
9. Una nueva realidad en el punto de partida.

Determinados grupos de investigadores han llegado a conclusiones muy drásticas: «en general, los métodos utilizados hasta la fecha no incluyen evaluaciones de las posibles consecuencias sociales y de salud pública acarreadas por el consumo de drogas, ni son apropiados para hacerlo» (Comité de Expertos, 1981); posición que es algo exagerada, ya que si bien el peso de la percepción social ha condicionado el diseño de las investigaciones, impidiendo el acceso a los problemas reales, eso no significa que las metodologías no valgan.

Los mismos autores establecen que para los fármacos psicotrópicos los problemas que hay que valorar son: problemas de comportamiento, efectos sobre la conducción de vehículos, psicomotricidad, actividades delictivas e ilegales, efectos socioeconómicos, etc. (Comité de Expertos, 1981), sobre los que ya se han realizado evaluaciones (sólo hay que recordar el tema de la conducción de vehículos) con los métodos estándar.

Las dificultades en la identificación de los problemas proceden de la falta de toma en consideración de otra realidad: desde la medida de la amplitud del uso de las distintas drogas hasta el análisis causal y la caracterización de su uso, dependen de la concepción sociocultural del investigador. La determinación del tipo de medida es siempre sociocultural; por ejemplo, la discusión sobre el consumo medio diario de alcohol puro que es peligroso para la salud no es más que el reflejo de las posiciones contrapuestas entre los banalizadores sociales y los partidarios de una conceptualización metabólica del problema; así se explica que haya estudios con la barrera puesta en 20 c.c. y otros en 150 c.c. Otro ejemplo podría ser la tendencia en calcular sólo los problemas relacionados con ansiolíticos a partir de que el usuario cumpla la condición de consumirlos o no con receta médica, como si el acto administrativo médico modificara los efectos sobre la salud.

Esta realidad ha mediatizado absolutamente todas las investigaciones y resulta evidente que hay que evitarla, pero teniendo también en cuenta que el uso de drogas produce dos tipos de consecuencias socioculturales: las unas funcionales, las otras disfuncionales, y que las primeras implican una serie de estereotipos variables pero inevitables y nunca tenidos en cuenta.

«Separar cualquier aspecto de la respuesta terapéutica de sus determinantes y su contexto socioculturales equivale a considerar el Tótem o el objeto fetiche como si fueran solamente objetos de arte abstracto.»

«Puede, en verdad, parecer extraordinario que se haya escrito tanto hasta la fecha sobre la Sociología y la Antropología del consumo de drogas y que, al mismo tiempo, se haya hecho tan poco para aplicar estas ciencias al estudio de los orígenes sociales y culturales de la respuesta terapéutica» (Edwards, 1981). 
Esta carencia se articula sobre la creencia de que es posible obtener, en el campo del fenómeno del uso de drogas y las drogodependencias, conocimientos sin mediación sociocultural. Esto es al menos discutible, en tanto la intervención es parte del fenómeno a estudiar, y la eficacia de ésta depende del grado de implicación sociocultural de los diferentes actores.

Observemos las consecuencias éticas de incluir en un mismo fichero de notificaciones o en un registro datos personales, judiciales y sanitarios en relación a unas conductas cuyo status legal es, al menos, ambiguo y que al ser identificadas producen necesariamente un proceso de etiquetado y desviación. La obtención del dato produce así consecuencias fundamentales sobre el fenómeno y llega a caracterizarlo. ¿Se llegan a preguntar en alguna ocasión los autores del DARP, DAWN, CODAP, TOPS, AI, etc, por la estereotipación social que producen sus sistemas? Desde luego, nunca, en ningún caso, bajo ningún concepto.

¿Cómo resolver esta situación? ¿Cómo desarrollar investigación controlando la retroalimentación que producen sobre el fenómeno? ¿Cómo evitar interpretaciones sesgadas socioculturalmente? Preguntas que sólo podemos resolver a través de una dinámica que integra, por una parte, todas las posibilidades metodológicas y, por otra parte, sea consciente permanentemente de sus limitaciones y mediaciones socioculturales.

En este sentido es preciso desarrollar los trabajos que contemplen tales factores con metodologías que se orienten hacia el aislamiento de los factores más subjetivos del fenómeno.

Tomemos como punto de partida la resolución del problema del secreto, en una conducta expresiva, que ya hemos mencionado antes, y que es muy relevante en las relaciones de compraventa o uso de drogas ilegales, introduciendo dificultades en la realización de las encuestas.

Este secreto varía en función de las características de la legislación penal, de las características socioeconómicas y sociodemográficas de los usuarios y el tipo de droga utilizada, y condiciona una determinada estrategia de secreto, habiéndose descrito modelos de la misma (Hughes, 1982).

A partir del conocimiento de tales modelos existen métodos etnográficos basados en la técnica combinada de la «zona blanco» (lugar donde se compra, se vende o se usa una droga ilegal) y de «bola de nieve» (seguimiento personalizado de los compradores o usuarios de la «zona blanco»); proporciona una «muestra» relevante de usuarios completamente distinta a la «muestra» de usuarios atendidos (Medina-Mora, 1980). A esta «muestra» se le pueden aplicar diversas metodologías desde cuestionarios a historias de vida.

Las dificultades residen aquí en una adecuada selección del equipo de trabajo de campo; su nivel de preparación, edad y características físicas determinan los posibles contactos y las posibilidades de obtener información.

Así se explica que se puedan obtener conclusiones muy claras con estos métodos y un cuestionario como el «Formulario Marmottan» (Ingold, 1984) 
nos llegue a proporcionar mayor información que todos los sistemas de notificación y los diversos indicadores. Información y conclusiones no representativas, quizá, pero que valen para completar el mapa que nos pueden proporcionar otras metodologías.

Para acabar sintetizando una posición y abriendo un punto de partida nuevo, vale la pena exponer extensamente la técnica de la cadena de referencia, en el caso concreto del análisis de las redes sociales de cocaína (Kaplan, 1986).

El problema metodológico de la cocaína resulta conocido: se está incrementando considerablemente su uso al tiempo que se están modificando los hábitos de consumo con la aparición de la vía endovenosa. Tenemos acceso al conocimiento de esta realidad a través de datos como el incremento de las aprehensiones, el número de experimentadores que aparece en las encuestas y la observación directa, pero los sistemas de notificación no nos permiten acceder a la incidencia, prevalencia y problemas asociados al uso de cocaína. Se trata de una actividad ilegal cuya incidencia sobre la salud pública no se manifiesta por muertes ni por sobredosis, ni en los servicios de urgencia hospitalarios.

Para obviar estos problemas, Kaplan propone desarrollar una nueva metodología que, en sí, puede constituir un «indicador técnico de la incidencia y prevalencia de cocaína». El punto de partida es la experiencia obtenida con la técnica de la acumulación de dificultades imprevistas que se ha utilizado para la heroína, seleccionando individuos altamente criminalizados y sometiéndoles a un proceso de control permanente de actividades durante el período de investigación.

Esta técnica permitió entender que el uso de cualquier droga ilegal implica algún tipo de actividad social, aunque sea el simple acto de la compraventa, que puede ser medida y cuantificada, a través de una cadena de referencia que sale y acaba en el último consumidor.

Por otra parte, el análisis de las redes sociales permite identificar matemáticamente los nudos donde confluyen los eslabones que configuran un fenómeno social.

La combinación de las técnicas de la cadena de referencia y el análisis de las redes sociales permite obtener una muestra representativa de la población usuaria de cocaína. En una investigación-piloto, Kaplan va a recoger datos de tres redes distintas de usuarios de cocaína en cada una de las ciudades seleccionadas: un estrato de profesionales de clase media, un estrato de estudiantes e intelectuales y un tercer grupo de adictos a la heroína. Cada red va a tener de 10 a 20 individuos, con lo que la muestra-piloto se va a hacer entre 90 y 180 individuos, a los que se les va a pasar un cuestionario muy completo.

De todas maneras, se pretende que no todos los individuos contactados 
a través de la red sean entrevistados, sino únicamente los seleccionados con técnicas aleatorias.

Se prevé que factores como el nivel socioeconómico de la familia, el grado de implicación personal, el nivel de estudios, la red de influencias, etc., determinen la estructura y amplitud de la red, así como el tipo específico de consumidor y su nivel de presencia sobre la extensión de la variable. A partir de ahí será posible un resultado cuantitativo sobre incidencia y prevalencia, utilizando en el análisis el supuesto de la similitud entre variables descriptivas.

En resumen, un trabajo de campo etnográfico sobre cuyo desarrollo se introducen controles estadísticos y de representatividad y que, finalmente, nos va a proporcionar unos datos que elevamos a la categoría de panel, nos conforman un verdadero indicador de la evolución de la incidencia, prevalencia y problemas asociados al uso de cocaína.

\section{BIBLIOGRAFIA}

Alonso Torrens, F. J., et al.: La población española ante las drogas, Madrid, Documentación Social, 1981.

BACHMAN, Jerald G., et al,: «Drug use among young adults: the impacts of role status and social environment», Journal of Personality and Social Psychology, vol. 47, núm. 3, 1984.

Balter, M. B., et al.: «Cross National Studies of the extent of antianxiety/sedative drug usew, New England Journal of Medicine, núm. 290, 1974.

Comas Arnau, Domingo: El uso de drogas en la juventud, Madrid, Ministerio de Cultura, 1985.

- Panorama y alternativas metodológicas en el estudio de los aspectos sociales y culturales del consumo de drogas en España, Zaragoza, I Congreso de Sociología, MINEO, 1981.

Comité DE EXPERTos: Evaluación de los problemas sociales y de salud pública relacionados con el uso de sustancias psicotrópicas, Ginebra, Organización Mundial de la Salud (OMS), 1981.

Crider, Raquel, et al.: Heroin Indicators Trend Report. An update 1976-78, Rockville, NIDA, 1979.

Davidson, F., et al.: «Recherche d'une typologie des jeunes toxicomanes», Psycbiatrie Entant, vol. XXV, núm. 2, 1982.

Díaz DE Quijano, S., et al.: Los Servicios de atención a toxicómanos, Madrid, Dirección General de Acción Social-Cruz Roja, 1983.

Driessen, F. M. H. M.: The use of Alcobol and drugs among pupils in secondary education and international comparative study, Amsterdam, MINEO, 1985.

Edwards, G., y ARIF, A.: Los problemas de la droga en el contexto sociocultural, Ginebra, OMS, 1981.

Feldman, Harvey W., y Waldorf, Dan: Angel Dust in four American Cities: An Etbnograpbic study of PCP users, Lexington, U. S. Department of Health and Human Services, 1980.

Guardia, J., y GonzÁlez, S.: «Estudio de seguimiento (un año) de adictos a la heroína», Revista del Departamento de Psiquiatria de la Facultad de Medicina de Barcelona, vol. X, núm. 6, 1983. 
Hug i Es, P. H., et al.: «Modelos etnográficos y de secreto entre los toxicómanos», Bo. letin de Estupefacientes, vol. XXXIV, núm. 1, 1982.

- L'usage des drogues à des fins non médicales: données de base en vare des études épidémiologiques, Gincbra, OMS, 1983.

INGOLD, R., y OLIVENSTEIN, C.: "Conclusiones preliminares de una encuesta epidemiológica sobre toxicomanía en París», Boletin de Estupefuctentes, vol. XXXV, núm. 3, 1983; Nueva York, 1984.

Jon, George W., y GrNT. Michael: Drug treatment bistories for a sample of drug users in DARP, Rockville, NIDA, 1978.

Johnston, Lloyd D.: Review of General Population strveys of drug abuse, Ginebra, OMS, 1980.

Jo hanton, Lloyd D., y O'Mari.ex, Patrick M.: And Assessment of student surveys as a technique for gatbering epidemiological data on drug use, Ann Arbor, MINEO, 1984.

JoHnston, Lloyd D., et al.: Use of licit and ilicit drugs by America's bigh scbool students 1975-1984, Rockville, NIDA, 1985.

- Drugs and American bigh school students 1975-1983, Rockville, NIDA, 1984.

KAPLAN, C. D.: The theory of referral chains for Cocaine Nelworks, Luxemburgo, EEC, 1986.

Kozel, Nicholas I.: Heroin Indicators Trend Report 1976-1978. An update, Rockville, NIDA, 1978.

- Epidemiology of Heroin. 1964-1984, Rockville, NIDA, 1985.

LinvBl.AD, R. A.: «Estudio sobre el movimiento de padres contra el uso indebido de drogas en los Estados Unidos de América», Boletin de Estupetacientes, vol. XXXV. núm. 3, 1983.

Mrdina-Mora, M. E., et al.: «Metodología para la identificación intensiva de casos y la vigilancia del uso de drogas en una comunidad mexicanas, Boletin de Estupefacientes, vol. XXXII, núm. 2, 1980.

- «Validez y fiabilidad de un cuestionario utilizado para estudiar el uso de drogas entre estudiantes mexicanos de Enseñanza Media», Boletin de Estupefucientes, vol. XXXIII, núm. 4, 1981.

Ministerio de. Sanidad y Consumo: Plan Nacional sobre Drogas, Madrid, 1985.

MoHAN, D., et al.: «Identificación de personas farmacodependientes en una encuesta: Nota sobre cuestiones de metodologia», Boletin de Estupefacientes, vol. XXXII, núm. 2, 1980.

Monegal. Ferriol, I. M., et al.: «Informe para un plan de lucha contra las farmacodependencias de tipo no alcohólicon, IX Congreso Internacional sobre Prevención y Tratamiento de las Drogodependencias, Madrid, INSERSO, 1980.

Mulder, Paul G. H.: Matbenatical modeling of risks of becoming (in) dependent, Luxemburgo, EEC, 1986.

Obiols VIF, J., et al.: "Nuestra experiencia en el tratamiento de heroinómanos», IX Congreso Internacional sobre Prevención y Tratamiento de las Drogodependencias, Madrid, INSERSO, 1980.

Person, Philip H., et al.: Touard a Heroin problem index. And analytical model for drug abuse indicutors, Rockville, NIDA, 1976.

- A metbod for estimating Heroin use prevalence, Rockville, NIDA, 1977.

Ramón Cavero, Miguel Angel, et al:: «Primeras impresiones sobre la población asistida en el Ambulatorio-piloto del Centro Asistencial de Drogras de la Cruz Roja Española», IX Congreso Internacional sobre Prevención y Tratamiento de' las Drogodependencias, Madrid, INSERSO, 1980.

Rodríguez-Martos, A., y Welsch, T.: «Psicología del toxicómano actual: Análisis crítico a partir del estudio protocolizado de 48 casos», Boletín del INSERSO, núm. 1, 1980.

Rootman, I., y Hughes, P. H.: Abus des drogues: Systemes de notifications, Ginebra, OMS, 1983.

Rootman, Irving, et al.: Normas para investigar los problemas relacionados con el alcobol y preparar las soluciones adecuadas, Ginebra, OMS, 1985. 
Rufener, B. L., et al.: Management efectiveness measures for NIDA. Drug abuse treatment programs, 2 vols., Rockville, NIDA, 1976.

RutTenber, James A., y LUKE, James L.: «Heroin-related deaths: New epidemiologic Insights», Science, vol. 226, octubre 1984.

SANTo, Yoav, et al.: Highlights from the National Youtb Polvdrug Study, Rockville, NIDA, 1980.

Sells, S. B., y Simpson, D. D.: «Eficacia del tratamiento del uso indebido de drogas: Resultados del programa de investigación DARP en los Estados Unidos de América», Boletin de Estupefacientes, vol. XXXI, núm. 1, 1979.

Seva Díaz, A.: Metodología en la investigación epidemiológica del alcobolismo, Zaragoza, Universidad de Zaragoza, 1984.

Simon, C. P., y WiTte, A. D.: Beating the system. The underground Economy, Boston, Auburn House, 1982.

SMART, R. G.: L'usage des drogues cbez les jeunes non-étudiants, Ginebra, OMS, 1981.

S Mart, R. G., y Murray, G. P.: «Examen de las tendencias del consumo de alcohol y de cannabis entre los jóvenes», Boletín de Estupefacientes, vol. XXXIII, núm. 4, 1981; Nueva York, 1982.

Smart, R. G., et al.: A methodology for student drug-use surveys, Ginebra, OMS, 1980.

Tims, F., y Ludford, J. P. (eds.): Drug abuse treatment evaluation: Strategies, progress and prospects, Rockville, NIDA, 1984.

Vila-Abadal, J.: «Aspectes psicopatológics de 115 heroinomans visitats a spott», I Jornades Catalanes de Sociodrogalcohol, Barcelona, 1983. 